\title{
DIVISION I: FUNDAMENTAL ASTRONOMY
}

\author{
(ASTRONOMIE FONDAMENTALE)
}

\author{
PRESIDENT: Nicole Capitaine \\ BOARD: Jean Chapront, John D. Hadjidemetriou, Wenjing Jin, Gérard Petit \\ \& Kenneth Seidelmann
}

\author{
Commission 4: Ephemerides \\ Commission 7: Celestial Mechanics and Dynamical Astronomy \\ Commission 8: Astrometry \\ Commission 19: Rotation of the Earth \\ Commission 31: Time
}

\section{Introduction}

This report includes the highlights of each commission belonging to Division 1 and the reports of each Division 1 Working Group for the triennium 1999-2002.

Some general developments of particular interest deserve special mention.

New resolutions have been adopted during the General Assembly in Manchester among which resolutions B1 and B2 are of special interest to Division I; they have been prepared and discussed at IAU Colloquium 180 in Washington (March 2000) and then discussed again at Joint Discussion 2 "Models and Constants for Sub-microarcsecond Astrometry" during the GA. Resolution B1 provides definitions, standard algorithms and parameters for modern realisations and transformations of space and time reference systems within the framework of General Relativity; this resolution, which is based on the adoption of the International Celestial Reference System (ICRS) as the IAU celestial reference system, from 1 January 1998, results from the proposals of Working Groups of the Division. Resolution B2 recommends to investigate possible changes in the current definition of UTC, which concerns especially time and Earth rotation studies. During the triennium 1999-2002, Working Groups of Division I have been organized to implement these resolutions.

In order to follow the evolution of astrometry, the merger of Commissions 8 "Positional Astronomy" and 24 "Photographic Astrometry" into one new Commission 8 "Astrometry" has been achieved at the GA in Manchester.

The International Earth Rotation Service (IERS), which was created in 1988 by the IAU and the IUGG, has got a new structure since January 1,2001, corresponding to new Terms of Reference. IERS is now composed of a Central Bureau, six Product Centres, ten Combination Research Centres and the Analysis Coordinator. External services like IGS, ILRS and IVS serve as Technique Centres for the IERS. A new Directing Board has been formed (Chair: Jan Vondrák) and the new web site of the IERS, http://www.iers.org, provides key information on the IERS and its components as well as links to the IERS components.

The International VLBI Service for Geodesy and Astrometry (IVS), which was created on 1 March 1999 as a Service of IAG and has been recognised as an IAU Service Organisation at the last General Assembly in Manchester (Resolution B1.1), has now become, in 2001, a new member of the Federation of Astronomical and Geophysical Data Analysis Services (FAGS). 


\section{Highlights}

\subsection{Ephemerides (Commission 4)}

Jean Chapront, Pres. Jet Propulsion Laboratory (Caltech) has built the planetary and lunar ephemeris DE405 and its extended version DE406 which covers 6 millennia. These export ephemerides are available on $\mathrm{CD}$ and form the basis of several international Almanacs. A similar construction has been undertaken at the Institute of Applied Astronomy (IAA, St Petersburg) which generated new numerical ephemerides EPM2002 for the major planets and Moon, including also a large number of asteroids. Concerning the lunar part of the ephemerides based on Lunar Laser Ranging, a simultaneous effort has been done by JPL, IAA and Paris Observatory (Paris Observatory Lunar Analysis Center). Several institutions have developed electronic access to data : Website and FTP server containing on line ephemerides, in particular for natural satellites at the Institut de Mécanique Céleste et de Calcul des Ephémérides, IMCCE - Bureau des Longitudes; Astronomical Almanac on line produced by Her Majesty's Nautical Almanac office of the UK and the U.S. Nautical Alamanac Office; data for apparent places of fundamental stars provided via internet by the Astronomishes Rechen-Institut.

\subsection{Celestial Mechanics and Dynamical Astronomy (Commission 7)}

John Hadjidemetriou, Pres. During the period 1999-2002 research has been focused in the following subjects: (i) Analytic studies and numerical integrations of Near Earth Objects, (ii) Study of the stability of extrasolar planetary systems, (iii) Long term stability of asteroids, including the Trojan asteroids, (iv) Effect of nongravitational perturbations, mainly the Yarkovsky effect, i.e. the recoil force of the thermally re-radiated sunlight by the surface of a cosmic body, (v) Theory and techniques for the detection of potential planetary impacts.

During the above period two meetings on Celestial Mechanics were held, where all the above topics were discussed: The Fifth Alexander von Humbolt Colloquium on Celestial Mechanics, Badhofgastein (Austria), 19-25 March, 2000 and the third Meeting on Celestial Mechanics, CELMEC III, MontePorzio, Roma, 18-23 June, 2001.

\subsection{Astrometry (Commission 8)}

Wenjin Jin, Pres. With the success of the Hipparcos mission and the release of the Hipparcos and Tycho Catalogues, many astronomers are involved in the preparation of secondgeneration astrometric satellites to achieve the micro-arcsecond precision. In the last three years great progress has been made in such active space astrometry programmes as DIVA, GAIA, SIM and FAME.

$\mathrm{CCD}$ is a powerful device in current astrometric observations. A three-year monitoring campaign for WIYN open cluster program, used the NOAO CCD Mosaic Imager at Kitt Peak. The projects of extension and maintenance of Hipparcos catalogue have been carried out. With the improvements of the CCD performance, catalogues containing more stars and limited magnitude up to $16-17$ mag. were distributed. The catalogues combining the old photographic plates or the earlier catalogues with the current observations were released to improve the proper motions. Meantime the comprehensive data banks ARIGH and WFPDB for astrometric data of the stellar positions and proper motions and the photographic plates were set up respectively. The optical positions of extragalactic radio or infrared sources were determined for the link between extragalactic reference and reference frame in optical or other wavelength and the catalogues of second reference stars were published. The joint projects for link of references between observatories such as USNO and Hamburg Observatory; Kazan, Shanghai, Turkey and Nikolaev Observatory are implemented. The international Campaigns for network observing the planets and their satellites were carried out and the PHEMU 2003 has been prepared for ephemeris development. Besides the 
ground-based observations, the mean motions for four inner satellites of Neptune have been determined by HST.

Applications of astrometric parameters on astrophysical and kinematical studies of open and globular clusters, galactic rotation, kinematics of various stellar populations etc. are becoming important branches of astrometry. The astrometric observations focus on the sky area for astrophysical interests such as the pre-main sequence stars of star-forming regions. A substantial, directly observed galactic halo dark matter, the population of old white dwarfs, too faint to be seen in previous surveys is found in the data of SuperCOSMOS sky survey which accounts for at least $2 \%$ of the halo dark matter. A joint investigation has examined the potential to detect flare stars from triple images found in the 130-year-old Bordeaux, Brussels and Uccle astrographic plates. A sample of 200 variables found that possibly $10 \%$ are pre-main sequence stars.

\subsection{Rotation of the Earth (Commission 19)}

Nicole Capitaine, Pres. During the triennium 1999-2002, a complete reorganization of the International Service in charge of monitoring Earth rotation has taken place. Such a reorganization has been accompanied by significant improvements in both theory and observations of Earth rotation and by the preparation of the implementation of the IAU Resolutions 2000. These resolutions, which will become effective on January 1, 2003, imply the adoption of a new precession-nutation model (IAU 2000), of a new celestial pole (the Celestial Intermediate Pole) and of a new transformation between the terrestrial and celestial systems which defined UT1 as directly proportional to the Earth Rotation Angle. Most of the results obtained during this period have been derived from a combined use of models and observations. This has significantly improved the model for the nutation of non-rigid Earth and the understanding of the influence of the atmosphere, oceans and core on length-of-day changes and on the excitation of Chandler wobble.

\subsection{Time (Commission 31)}

Gérard Petit, Pres. The relative frequency stability and the accuracy of atomic time scales, like International Atomic Time TAI, is now of order $1 \times 10^{-15}$ thanks to progresses in clock technology and in clock comparison techniques. Cold atom primary Cs standards have a stated accuracy of $1 \times 10^{-15}$ and a stability in the $10^{-16}$ region. Other cold atom clocks provide even better prospects, as well as clocks based on trapped ions. Frequencies based on optical and microwave transitions can now be compared with a similar or even better uncertainty thanks to femtosecond comb technology. Clock comparison techniques based on GPS (see http://maia.usno.navy.mil/gpst.html), or on dedicated Two Way technology provide adequate performance when averaging data over one or a few days, and should be improved to accompany the progresses of clocks. Since 1999, a number of organizations initiated a review on the future of the UTC system (insertion of leap seconds between TAI and UTC to keep $|U T 1-U T C|<0.9 \mathrm{~s}$ ). Several working groups have been initiated, notably by the International Telecommunications Union (Special Rapporteur Group (SRG) in the Working Party 7A), by the International Union of Radio Science, and by the IAU following Resolution B2(2000). No immediate conclusion may be foreseen but a consensus should be reached over the next triennium.

\section{Working Groups}

\subsection{Working Group on International Celestial Reference System (ICRS)}

\section{François Mignard, Chairman}

Creation and Organization

The Working Group on the Celestial Reference System was renewed at IAU General Assembly XXIV in August 2000 for a term of three years, with the goal of coordinating 
the work of astronomers to qualify, use, extend and promote the ICRF and prepare the recommendations relevant to these topics to be submitted to the IAU General Assembly in 2003. The Working Group comprises 39 members and is organized around six well identified tasks directed by a task leader. Each member of the working group has expressed personal interest in at least one of these task. The WGICRS was created as a Working Group of IAU

Table 1. Tasks and task leaders of the Working Group

\begin{tabular}{lll}
\hline Task & Label of the task & Chair \\
\hline $\mathbf{T}_{1}$ & Maintenance and extension of the ICRS & C. Ma \\
$\mathbf{T}_{2}$ & Densification at optical and IR wavelengths & S. Urban \\
$\mathbf{T}_{3}$ & Space Astrometry and Reference Frames & F. Mignard \\
$\mathbf{T}_{4}$ & Link to the dynamical system & M. Standish \\
$\mathrm{T}_{5}$ & Computational tools & P. Wallace \\
$\mathbf{T}_{6}$ & Astronomical Standards & T. Fukushima \\
$\mathbf{T}_{6}$ & Relation with IERS & F. Arias \\
\hline \hline
\end{tabular}

Division I as a continuation of similar groups since 1991 whose activities led to the adoption of the ICRF as a realization of the ICRS in 1997 and to an extensive set of resolutions in 2000 which provides the general framework of modern astrometry.

Main activities 2000-2002

(i) Densification

The densification is the area where the most significant advances took place during this period. For all-sky catalogues, Tycho-2 Catalogue (Hoeg et al. 2000), utilizing re-reduced Tycho star mapper data from the Hipparcos satellite and over 140 groundbased observational catalogs for the computation of proper motions, was released by Copenhagen University Observatory and the U.S. Naval Observatory in February 2000. For deeper catalogs, the Guide Star Catalogue 2.2 with 435 million stars was made available via the web www-gsss.stsci.edu/gsc/gsc2 in 2001. This, along with the USNO A2.0 (released 1998) of 526 million stars, are the current standards for stars fainter than those found in Hipparcos or Tycho-2. Regarding zonal catalogue the first release of the USNO CCD Astrograph Catalog (UCAC1) was made in April 2000 (Zacharias et al. 2002). This catalog contains positions and motions for 27 million Southern Hemisphere stars in the magnitude range $\mathrm{R}=7.5$ to about 16.0. It should be followed by a second release near the end of 2002 covering more than half of the Northern hemisphere. The twelfth installment from the Carlsberg Meridian Telescope (Evans 2001) is now available (CMC12, version 1.0). This contains positions of 6.3 million stars in the magnitude range 9 to 17 in a six degree band centered on the equator. The M2000 catalogue has been released by the Observatoire de Bordeaux (Rapaport et al. 2001). It contains 2.3 million stars between +11 and +18 degrees, each observed about 6 times with the Bordeaux automated meridian circle over the past four years. In the near infrared realm, the Two Micron All Sky Survey (2MASS) released a second data set in 2000 , bringing the total sky coverage to about $50 \%$. Final data release covering the entire sky is expected by the end of 2002.

(ii) Space astrometry missions

The planned space astrometry missions should have a major impact on the realization of the ICRS, bringing it back into the visible range. However, after having selected 
FAME in October 1999, NASA withdrew its support in January 2002. The situation of the German mission DIVA is not much brighter at the moment and rather unpredictable. After a period of doubt, GAIA was confirmed in May 2002 as one of the ESA major missions for the beginning of the next decade. Simulations have shown that GAIA should be able to realize a fully optical primary frame with a residual rotation below $1 \mu$ as per year. But due to the canceling of the intermediate missions and the long delay before the launch of GAIA, the baseline of the ICRF will not change before at least 15 years.

(iii) Maintenance of the ICRF

The first extension of the ICRF was released in 1999, by incorporating VLBI sessions up to April 1999. 59 new sources not included in the initial ICRF were included and the new axes have no rotation with respect to the original system. A second extension is in preparation. The stability of the system has been investigated (Gontier et al. 2001) from the time series of the coordinates of the best observed sources, yielding a global stability of the axes of $4 \mu$ as over six years.

Using VLBA observations between 1994-1997, the VLBA Calibrator Survey (VCS1) generated 1300 new astrometric positions with declinations above -30 deg consistent with the ICRF.

Reference: Beasley, A.J., Gordon, D., Peck, A.B., Petrov, L., MacMillan, D.S., Fomalont, E.B., and Ma, C. 2002, Ap. J. Sup. Ser. 141:13-21.

(iv) Computing tools and astronomical standards

The main action to provide a standardized set of computing tools started in 1997 and the first release of a set of software and subroutines (Wallace 2000) was released in April 2001, when a collection of Fortran subroutines was published on the SOFA website http://ww. iau-sofa.rl ac.uk. This initial release consisted of a set of 52 general-purpose routines for handling vectors, matrices and angles, together with a small set of astronomical routines.

A major milestone in the implementation of the IAU 2000 resolution took place in 2002 with the organization of a very successful IERS workshop in Paris fully dedicated to all the practical aspects arising from these resolutions.

During this Workshop Wallace (2002) confirms his plans to implement the IAU 2000 resolutions in the next release to be made available by 1st January 2003. Extensive tests have been carried out by P.T. Wallace and N. Capitaine to ensure that the various ways of calculating the position of the CIP and the rotation of the Earth are equivalent, at the level of a few $\mu$ as.

v) Link to dynamical systems

The position of the inertial dynamical ecliptic with respect to the ICRS has been obtained from a new processing of the complete set of LLR observations (Chapront 2002). This new adjustment leads also to a correction to the precession constant in good agreement with the determination based on VLBI data.

\section{References:}

Chapront, J,, Chapront-Touzé, M. and Francou, G. 2002, A\&A, 387, 700.

Evans, D.W., 2001 Astronomische Nachrichten 332, 347

Gontier, A.M., Le Bail, K., Feissel, M., 2 - T. M. Eubanks, T.M. 2001, A\& A 375, 661.

Hoeg, E., Fabricius, C., Makarov, V.V., Urban, S., Corbin, T., Wycoff, G., Bastian, U., Schwekendiek, P., and Wicenec A. 2000, A\& A, 355, L27

Rapaport, M., De Campion, J-F., Soubiran, et al. 2001, A\& A 376, 325

Seidelmann, P. K.; Kovalevsky, J. 2002, A\& A, 392, 341. 
Wallace, P.T., 2000, "SOFA Progress Report", Proc. IAU Colloquium 180, eds. K.J. Johnstone, D.D. McCarthy, B.J. Luzum, G.H. Kaplan, USNO 353.

Wallace, P.T., 2002, in the Proceedings of the IERS Workshop, 5.1, Paris, April 2002, ww. iers.org/iers/publications/tn/tn29.

Zacharias, N., Urban, S.E., Zacharias, M.I., Hall, D.M., Wycoff, G.L., Rafferty, T.J., Germain, M.E., Holenried, E.R., Pohlman, J.W., Gauss, F.S., Monet, D.G., Winter,L., 2000 AJ 120, 2131.

\subsection{Working Group on General Relativity in Celestial Mechanics, Astrometry and Metrology (RCMAM)}

Michael Soffel, Chairman After the last IAU GA in Manchester and the adoption of the new IAU 2000 resolutions concerning relativity the work of the WG focused upon the realization and practical use of these resolutions. The role of the two Celestial Reference Systems, BCRS and GCRS, and of astrometric places of stars (e.g., apparent places) was discussed. Further points of intensive discussions were: the coordinate transformations between the BCRS and the GCRS, the mass multipole moments (potential coefficients) and especially the spin moments. Such spin moments, related with the rotation of the Earth play no role in Newton's theory of gravity. Orders of magnitude of these spin moments of the Earth were derived in the framework of a model. Further work concerns the implementation of these resolutions in the various models for VLBI, LLR, SLR etc. and in the IERS conventions.

A detailed explanatory supplement concerning these resolutions was prepared for publication.

\subsection{Working Group on Redefinition of Universal Time Coordinated (UTC)}

Dennis D. McCarthy, Chairman The IAU Working Group on the Definition of Coordinated Universal Time was created in compliance with IAU Resolution B2 of the XXIVth IAU General Assembly. The members are F. Arias, W. Dick, E. Fedoseev, D. Gambis, W. Klepczynski, S. Leschiutta, J. Luck, Z. Malkin, D. Matsakis, P. Paquet, J. Vondrák, P. Wallace, and S. Ye. D. McCarthy serves as Chairman of the Group and as IAU representative to the International Telecommunications Union (Radiocommunications) (ITU-R) Special Rapporteur Group (SRG) on the Definition of Universal Time.

The ITU-R has assumed international responsibility for the definition of UTC and the Special Rapporteur Group reports to the ITU-R with recommendations regarding possible future changes to the current procedure relating UTC to International Atomic Time (TAI). Two meetings of the ITU-R Special Rapporteur Group have been held. The first was held in Geneva, 3-4 May 2001 and the second meeting of the ITU-R Special Rapporteur Group met in Paris 21-22 March 2002.

The Special Rapporteur Group has converged to the opinion of freezing the present difference between UTC and International Atomic Time (TAI) at the current value of 32 seconds. It was decided at the Paris meeting that it would be necessary to retain the name "Coordinated Universal Time" and the abbreviation (UTC) to avoid potential problems regarding the definition of national time scales. UTC is the legal basis for time in many countries. Consequently, many laws might have to be rewritten to account for this change. A concern is the fact that, by freezing UTC, there would be two identical scales, shifted by 32 seconds (UTC and TAI). The SRG decided that it would make no recommendation regarding TAI.

The SRG also decided to organize a symposium by the end of 2002 to solicit the input of different communities relative to the opinion to freeze the difference between UTC and TAI. 
A formal recommendation to the ITU-R has not yet been formulated. The SRG would prefer to produce a formal recommendation to the ITU after considering all possible disadvantages of the proposal.

Issues that will require further consideration were outlined at the Paris meeting. Foremost among these are issues related to the fact that adoption of such a proposal would allow the difference between UT1 and UTC to be unbounded. Specifically it will be necessary to consider (1) the definition of DUT1, and (2) future provision of UT1-UTC information to users.

Working Group activity has been carried on by electronic correspondence. There is no clear consensus of opinions from that correspondence yet. There does seem to be some agreement that those users who require a continuous time scale should consider the increased use of TAI and that the options need to be more carefully considered before a specific recommendation can be made.

\section{References:}

McCarthy, D., 2000, "Future Definition of UTC", in Proceedings of IAU Colloquium 180, Towards Models and Constants for Sub-microarcsecond Astrometry, K.J. Johnston, D.D. McCarthy, B.J. Luzum, G.H. Kaplan, eds., U.S. Naval Observatory, Washington DC, USA, pp. 363-371.

Nelson, R.A., McCarthy, D.D., Malys, S., Levine, J., Guinot, B., Fliegel, H.F., Beard, R.L., Bartholomew, T.R., 2001, "The leap second: its history and possible future", Metrologia, 38, pp. 509-529.

\subsection{IAU/IUGG/COSPAR Working Group on Cartographic Coordinates Ro- tational Elements of the Planets and Satellites}

Ken Seidelmann, Chairman The IAU/IAG Working Group on Cartographic Coordinates and Rotational Elements of the Planets and Satellites report of 2000 was published in Celestial Mechanics and Dynamical Astronomy 82: 83-110, 2002. The authors are P.K. Seidelmann, V.K. Abalakin, M. Bursa, M.E. Davies, C. DeBergh, J.H. Lieske, J. Oberst, J.L. Simon, E.M. Standish, P. Stooke, and P.C. Thomas. The working group is reformulated for this triennium and preparing the next report in its continuing series. A draft of the report will be presented for discussion and completion at the IAU General Assembly in Sydney, Australia, in 2003.

\subsection{Working Group on Future Developments in Ground-Based Astrometry}

Jean Kovalevsky, Magda Stavinschi, Co-chairs In its last General Assembly (Manchester, August 2000), the IAU has set up a Working Group on The Future Development of Ground-based Astrometry to suggest scientific programs that can still be successfully performed with such instruments. It is headed by Magda Stavinschi (Romania) and Jean Kovalevsky (France). The members are: Daffy Wyn Evans (UK), Carlos Lopez (Argentina), Dan Pascu (USA), Antonio Pugliano (Italy), Manuel Sanchez (Spain), Ramachrisna Teixeira (Brazil), Arthur Upgren (USA). Consultant members and subgroups are: Alexandre Humberto Andrei (Solar diameter), Jean-Eudes Arlot (Mutual phenomena), Wim Brouw (Radio-astronomy), Janet A.Mattei (Photometry), Gennady Pinigin (Reference systems), Theodore Rafferty (Double stars). The main activity of the group is reflected on the WG site http://www.astro.ro/wg.html.

The first meeting of the WG was held during JENAM 2001, in Munich. The success of the Hipparcos, and the perspective of future astrometric space missions bring serious problems to many observatories and Universities that possess small instruments and used to do research in astrometry. The JENAM Joint Discussion 3 was the first occasion to exchange experience and reflect on the best use of existing instruments (Report was published in the EAS Newsletter, Issue 22, December 2001, p.8-9, and the list of the communications 
presented are published in Astronomische Gesellschaft, Abstract Series No.18, 2001). The same Report could be found on http://ww. astro.ro/raport_wg.html. A report on "the Ground-Based Astrometry before GAIA and Diva" was presented during JENAM 2002 in Porto (M. Stavinschi).

The second meeting was held during the international colloquium "Journées 2002 . Systèmes de référence spatio-temporels" (Bucharest, 25-28 September 2002),

http://www.astro.ro/journees2002.html. Jean Kovalevsky presented a "Synthesis of Possible Programs Using Small Ground-Based Astrometric Insrtuments". Other communications were devoted to different programs or techniques, as the limit capabilities of ground-based optical astrometry instruments and to the international campaign PHEMU 2003 for the mutual phenomena of jovian satellites which just started. The participants concluded that their discussion could continue on the WG site and that the group has to be enlarged.

\section{Web Sites}

The division has maintained a web site with links to the IAU, Commissions, Working Groups and meeting websites. The address is: http://syrte.obspm.fr/iaudiv1.

Acknowledgments. The Organizing Committee of Division 1 as well as the Chairs of the Division I Working Groups are kindly acknowledged for their contribution to the work of the Division during the triennium 1999-2001.

Nicole Capitaine

President of the Division 